\title{
Lorentz violation, gravitomagnetism, and intrinsic spin
}

\author{
Jay D. Tasson \\ Physics Department, Carleton College, Northfield, MN 55057
}

(Dated: August 2012)

\begin{abstract}
A largely unconstrained set of relativity-violating effects is studied via the gravitomagnetic effect on intrinsic spins. The results of existing comagnetometer experiments are used to place constraints on two new combinations of these effects at the $10 \%$ level. We show that planned improvements in these experiments will make them competitive with the best existing sensitivities to this elusive class of relativity-violating effects. Prospects for measuring the conventional General-Relativistic gravitomagnetic effect are also considered.
\end{abstract}

\section{INTRODUCTION}

Our present understanding of nature at the most fundamental level relies heavily on Einstein's theories of Special and General Relativity. Lorentz symmetry, the invariance of the laws of physics under rotations and boosts, is a foundational assumption of both of these theories. Testing such fundamental symmetries strengthens the experimental foundation of existing theories and offers the opportunity to detect hints of the elusive quantum-consistent theory at the Planck scale [1].

The gravitational Standard-Model Extension (SME) is a comprehensive theoretical framework for performing Lorentz-violation searches [2, 3]. The framework was constructed by adding all possible Lorentz-violating terms to know physics as described by the actions of the Standard Model of particle physics and General relativity. The Lorentz-violating terms involve coefficients for Lorentz violation, which can be measured or constrained experimentally. A large number of experimental results have been obtained in the context of the SME [4]. One class of experiments that has achieved impressive sensitivity has sought anomalous precessions of intrinsic spins in flat spacetime. Such experiments will be referred to as anomalous spin-precession experiments throughout this work. This class of experiments involves tests with macroscopic spin-polarized solids [5] and certain tests that can be thought of as clock-comparison tests [6] in which the frequencies depend on spin. As the level of sensitivity achievable in these experiments has evolved, they have become sensitive quantum gyroscopes [7, 8], detecting the fact that they are in a rotating reference frame attached to the Earth, and they have been reinterpreted as searches for spacetime torsion, placing the best constraints on constant background torsion [9]. These interpretations exploit the fact that these phenomena have the same coupling to intrinsic spin as the SME coefficients for Lorentz violation originally sought.

Gravitomagnetism is another effect with this same spin coupling [10]. As its name suggests, gravitomagnetism is a gravitational effect arising in analogy with classical electrodynamics [11]. Though the full theory of General Relativity is highly nonlinear, it is well known that the leading gravitational effects due to weak fields and slowmoving matter appear as analogues of the electric and magnetic fields of Maxwell electrodynamics. The relevant fields are known as the gravitoelectric and gravitomagnetic fields, and they generate an analogue of a Lorentz force on a moving test mass 12]. The analogy continues to spin precession, with gravitational fields generating a precession of angular momenta just as electromagnetic fields generate a precession of magnetic moments [13, 14]. This analogy has already been extended to the case of Lorentz violation in the pure-gravity sector of the SME 15], and the gravitomagnetic precession of classical angular momenta has now been observed [16].

In this work, we show that anomalous spin-precession experiments now have sensitivity to Lorentz-violating contributions to gravitomagnetism, which arise at lower post-newtonian order than the conventional gravitomagnetic effects of General Relativity. Two constraints on presently unconstrained combinations of coefficients for Lorentz violation stemming from both the pure-gravity sector 3, 17 and the gravitationally coupled matter sector [3, 10, 18] of the SME are achieved by reinterpreting the published results of comagnetometer experiments. One class of coefficients involved in this combination is difficult to detect, and with planned improvements in sensitivity, these experiments will be among the proposals 10] competitive with the best existing constraints on coefficients of this class.

In addition to placing constraints on Lorentz violation, we also explore the possibility of observing the conventional General-Relativistic gravitomagnetic effect on intrinsic spin using anomalous spin-precession experiments. The gravitoelectric effect on quantum particles was established by the experiment of Colella, Overhauser, and Werner (COW) [19] and is now observed routinely. Within General Relativity, as well as in most alternatives, the gravitomagnetic effect applies to intrinsic spin as well as to classical angular momenta, but establishing this experimentally would be of definite interest [20].

\section{BASICS}

The relevant theory is a special case of the gravitationally coupled SME [3] investigated in detail in Ref. [10]. In that work, the theory was treated perturbatively assuming weak gravity, asymptotically flat spacetime, slow- 
moving masses, and small Lorentz violation. As such, the Lorentz-invariant results of General Relativity and the flat-spacetime implications of Lorentz violation are included along with the leading Lorentz-violating modifications to gravity and can be recovered in the appropriate limit. Riemann-Cartan spacetime was considered as the geometrical framework allowing for a nonzero torsion, an addition warping of spacetime that can be considered in addition to the curvature of General Relativity.

The matter-sector SME coefficient for Lorentz violation $\bar{b}_{\mu}$, plays a key role in the analysis to follow. In the flat-spacetime limit of the SME, it enters the action in the form

$$
\mathcal{L}_{b}=-\bar{\psi} \bar{b}_{\mu} \gamma_{5} \gamma^{\mu} \psi
$$

making its spin coupling evident.

The relativistic hamiltonian relevant for the current discussion was obtained in Ref. [10]. The result contains a number of effects having couplings analogous to $\bar{b}_{j}$. These contributions can be written

$$
H \supset\left(-\widetilde{b}_{l}^{w}-\frac{1}{8} T^{\alpha \beta \gamma} \epsilon_{\alpha \beta \gamma l}+\frac{1}{4} \partial^{j} h^{0 k} \epsilon_{j k l}\right) \gamma_{5} \gamma^{0} \gamma^{l} .
$$

The first term here contains the following SME coefficients for Lorentz violation arising in the matter sector:

$$
\widetilde{b}_{j}^{w}=\bar{b}_{j}^{w}-\frac{1}{2} \epsilon_{j k l} \bar{H}_{k l}^{w}-m^{w}\left(\bar{d}_{0 j}^{w}-\frac{1}{2} \epsilon_{j k l} \bar{g}_{k l 0}^{w}\right) .
$$

The tilde notation denotes combinations of coefficients that arise together in the nonrelativistic expansion [4]. In general, the coefficients are particle-species dependent. The superscript $w=e, p, n$ indicates coefficients associated with electrons, protons, or neutrons respectively. The mass of the relevant particle is denoted $m^{w}$. The second term in the hamiltonian arises due to minimal torsion coupling to fermions. The presence of this term was exploited in Ref. 9], along with nonminimal torsion couplings to fermions, to place constraints on torsion using the results of anomalous spin-precession experiments. The third term in Eq. (2), is an effective $b_{\mu}$ containing gravitomagnetic effects. Here $h_{\mu \nu}$ is the metric fluctuation defined in terms of the spacetime metric and the Minkowski metric via the equation

$$
g_{\mu \nu}=\eta_{\mu \nu}+h_{\mu \nu} .
$$

Rotating frame effects also enter through this term when rotating coordinates are used. At the nonrelativistic level, the contributions in Eq. (2) lead to the hamiltonian contributions

$$
H_{\mathrm{NR}}=\left(-\widetilde{b}_{l}^{w}-\frac{1}{8} T^{\alpha \beta \gamma} \epsilon_{\alpha \beta \gamma l}+\frac{1}{4} \partial^{j} h^{0 k} \epsilon_{j k l}\right) \sigma^{l} .
$$

Numerous experiments have constrained $\widetilde{b}_{J}^{w}$ effects 4 , 7, 8, 21, 22]. The capital index here denotes constraints in the Sun-centered frame, which has been adopted as the standard frame for reporting sensitivities to SME coefficients in the context of flat-spacetime tests [23], and the concept has been extended to the post-newtonian limit
[17. At present, the most sensitive experiment investigating $\widetilde{b}_{J}^{e}$ is the spin-torsion pendulum at the University of Washington [8]. The pendulum bob consists of $\approx 10^{23}$ aligned electron spins while having negligible magnetic moment. The most sensitive experiments investigating $\widetilde{b}_{J}^{p}$ and $\widetilde{b}_{J}^{n}$ are a He/Xe comagnetometer [21] and a He/K comagnetometer respectively [7]. Comagnetometer experiments exploit the fact that Lorentz-violation couples to spin rather than magnetic moment. The colocated magnetometers can then be arranged such that signals from magnetic fields can be canceled while achieving impressive sensitivity to Lorentz violation. The constraints on $\widetilde{b}_{J}^{w}$ resulting from these experiments are summarized in Table I. Four orders of magnitude improvement over the $\widetilde{b}_{J}^{n}$ values listed in Table I are expected in the next generation of comagnetometer experiments [7].

Table I. Current order of magnitude sensitivities to $\widetilde{b}_{J}^{w}$.

\begin{tabular}{c|ccc}
\hline \hline & $w=e$ & $w=p$ & $w=n$ \\
\hline$\widetilde{b}_{X}^{w}$ & $10^{-31} \mathrm{GeV}$ & $10^{-31} \mathrm{GeV}$ & $10^{-32} \mathrm{GeV}$ \\
$\widetilde{b}_{Y}^{w}$ & $10^{-31} \mathrm{GeV}$ & $10^{-31} \mathrm{GeV}$ & $10^{-32} \mathrm{GeV}$ \\
$\widetilde{b}_{Z}^{w}$ & $10^{-29} \mathrm{GeV} \quad-\quad$ & -
\end{tabular}

For comparison with existing and proposed sensitivities to $\widetilde{b}_{J}$, it is convenient to define an effective coefficient for the gravitomagnetic effects entering Eq. (5):

$$
\left(b_{\mathrm{gm}}\right)_{l}=-\frac{1}{4} \partial^{j} h^{0 k} \epsilon_{j k l},
$$

where $h^{0 k}$ is understood to contain the contributions of interest for a given situation. Note that $\left(b_{\mathrm{gm}}\right)_{l}$ corresponds to the usual gravitomagnetic field in the General Relativity case.

\section{LORENTZ-VIOLATING EFFECTS}

We first consider Lorentz-violating contributions arising at second post-newtonian order. The post-newtonian metric associated with the coefficient for Lorentz violation $\bar{s}_{\mu \nu}$ appearing in the pure-gravity sector of the SME was obtained in Ref. [17]. A similar analysis was performed in the context of the gravitationally coupled matter sector in Ref. [10], obtaining contributions to the postnewtonian metric from coefficients $\left(\bar{a}_{\mathrm{eff}}\right)_{\mu}=\bar{a}_{\mu}-m \bar{e}_{\mu}$. For our present interest in lowest-order Lorentz-violating contributions, the off-diagonal elements the metric fluctuation appearing in Eq. (5) can be written

$$
h_{\mathrm{LV}}^{0 j}=\left[\bar{s}^{0 j}-\frac{\alpha}{m}\left(\bar{a}_{\mathrm{eff}}^{\mathrm{S}}\right)^{j}\right] U(x)+\left[\bar{s}^{0 k}-\frac{\alpha}{m}\left(\bar{a}_{\mathrm{eff}}^{\mathrm{S}}\right)^{k}\right] U^{j k}(x)
$$

in harmonic gauge. Here $\alpha$ represents a nonminimal coupling in the underlying theory of spontaneous Lorentzsymmetry breaking as discussed in Ref. [10]. The superscript $\mathrm{S}$ on the coefficient $\left(\bar{a}_{\mathrm{eff}}^{\mathrm{S}}\right)^{\mu}$ indicates that this is a composite coefficient associated with the particle content of the source of the gravitational field defined as

$$
\left(\bar{a}_{\mathrm{eff}}^{\mathrm{S}}\right)^{\mu}=\sum_{w} N^{w}\left(a_{\mathrm{eff}}^{w}\right)^{\mu}
$$


where $N^{w}$ is the number of particles of type $w$ contained within the source. The newtonian potential is denoted $U(x)$, and

$$
U^{j k}(x)=G \int d^{3} x^{\prime} \frac{\rho\left(\vec{x}^{\prime}, t\right) R^{j} R^{k}}{R^{3}},
$$

where $G$ is Newton's constant, $R^{j}=x^{j}-x^{\prime j}, R=\left|\vec{x}-\vec{x}^{\prime}\right|$, and $\rho\left(\vec{x}^{\prime}, t\right)$ is mass density.

Using Eqs. (6) and (7), one could in principle calculate the leading Lorentz violating contributions due to $\bar{s}_{\mu \nu}$ and $\left(\bar{a}_{\text {eff }}\right)_{\mu}$ in anomalous spin-precession experiments for any source. For the experiments considered here, Earth is the most relevant source. At the level of sensitivity available, it is sufficient to model it as spherically symmetric, with the local vertical in the lab (3 direction) pointing away from its center. Under these conditions we find the relevant contributions to Eq. (6) can be written

$$
\left(b_{\mathrm{gm}, a, s}\right)_{j}=\frac{1}{2} g \epsilon_{3 k j}\left[\bar{s}^{0 k}-\frac{\alpha}{m_{\oplus}}\left(\bar{a}_{\mathrm{eff}}^{\oplus}\right)^{k}\right],
$$

where $g$ is the local gravitational field of the Earth. The reader is cautioned that while the analogy between $\widetilde{b}_{j}$ and $\left(b_{\mathrm{gm}, a, s}\right)_{j}$ exists in the laboratory frame, there is not typically a direct match between the relevant coefficients for Lorentz violation in the Sun-centered frame due to the nontrivial dependence of $\left(b_{\mathrm{gm}, a, s}\right)_{j}$ on the coefficients $\bar{s}^{0 k}-\frac{\alpha}{m_{\oplus}}\left(\bar{a}_{\text {eff }}^{\oplus}\right)^{k}$. For example, for experiments performed on the surface of the Earth, the time-dependence of $\widetilde{b}_{j}$ can be displayed explicitly as

$$
\begin{aligned}
& \widetilde{b}_{1}=\widetilde{b}_{X} \cos \chi \cos \omega T+\widetilde{b}_{Y} \cos \chi \sin \omega T-\widetilde{b}_{Z} \sin \chi \\
& \widetilde{b}_{2}=-\widetilde{b}_{X} \sin \omega T+\widetilde{b}_{Y} \cos \omega T \\
& \widetilde{b}_{3}=\widetilde{b}_{X} \sin \chi \cos \omega T+\widetilde{b}_{Y} \sin \chi \sin \omega T+\widetilde{b}_{Z} \cos \chi(
\end{aligned}
$$

while for $\left(b_{\mathrm{gm}, a, s}\right)_{j}$ the time dependence takes the form

$$
\begin{aligned}
\left(b_{\mathrm{gm}, a, s}\right)_{1}= & \frac{1}{2} g\left[\bar{s}^{T X}-\frac{\alpha}{m_{\oplus}}\left(\bar{a}_{\mathrm{eff}}^{\oplus}\right)^{X}\right] \sin \omega T \\
& -\frac{1}{2} g\left[\bar{s}^{T Y}-\frac{\alpha}{m_{\oplus}}\left(\bar{a}_{\mathrm{eff}}^{\oplus}\right)^{Y}\right] \cos \omega T \\
\left(b_{\mathrm{gm}, a, s}\right)_{2}= & \frac{1}{2} g\left[\bar{s}^{T X}-\frac{\alpha}{m_{\oplus}}\left(\bar{a}_{\mathrm{eff}}^{\oplus}\right)^{X}\right] \cos \chi \cos \omega T \\
& +\frac{1}{2} g\left[\bar{s}^{T Y}-\frac{\alpha}{m_{\oplus}}\left(\bar{a}_{\mathrm{eff}}^{\oplus}\right)^{Y}\right] \cos \chi \sin \omega T \\
& -\frac{1}{2} g\left[\bar{s}^{T Z}-\frac{\alpha}{m_{\oplus}}\left(\bar{a}_{\mathrm{eff}}^{\oplus}\right)^{Z}\right] \sin \chi \\
\left(b_{\mathrm{gm}, a, s}\right)_{3}= & 0 .
\end{aligned}
$$

In spite of this subtlety, sufficient information exists to place constraints using published limits on $\widetilde{b}_{J}$. Note that the most sensitive investigations of $\widetilde{b}_{J}$ may be applied without regard to flavor, since the gravitomagnetic effects considered here are independent of test-body flavor.

Ignoring flavor, the best sensitivities to $\widetilde{b}_{X, Y}$ at present come from the $\mathrm{He} / \mathrm{K}$ comagnetometer experiment [7]. In that work, measurements were initially made of a magnetic field-like parameter $\beta^{N}$ with hamiltonian contribution $H \supset-\mu_{3} H_{e} \beta_{i}^{N} \sigma_{i}^{N}$, where the superscript $N$ denotes quantities associated with the nucleus, and $\mu_{3} \mathrm{He}$ is the magnetic moment of the ${ }^{3} \mathrm{He}$ nucleus. Measurements of

$$
\begin{aligned}
& \beta_{X}^{N}=(-0.020 \pm 0.040) \mathrm{fT} \\
& \beta_{Y}^{N}=(0.061 \pm 0.051) \mathrm{fT}
\end{aligned}
$$

were obtained based on investigation of the 1 direction, and consideration of the 2 direction yielded [24]

$$
\begin{aligned}
& \beta_{X}^{N}=(0.011 \pm 0.024) \mathrm{fT} \\
& \beta_{Y}^{N}=(0.025 \pm 0.022) \mathrm{fT} .
\end{aligned}
$$

These results were then combined and interpreted as constraints on $\widetilde{b}_{X, Y}^{n}$ in Ref. [7]. Here they can be used along with Eq. (12) to obtain the following results:

$$
\begin{aligned}
& \bar{s}^{T X}-\frac{\alpha}{m_{\oplus}}\left(\bar{a}_{\mathrm{eff}}^{\oplus}\right)^{X}=(0.24 \pm 0.15) \\
& \bar{s}^{T Y}-\frac{\alpha}{m_{\oplus}}\left(\bar{a}_{\mathrm{eff}}^{\oplus}\right)^{Y}=(0.02 \pm 0.13) .
\end{aligned}
$$

Here all uncertainties are 1 sigma.

With the expected improvements in the $\mathrm{He} / \mathrm{K}$ comagnetometer experiment [7], sensitivities at the level of $10^{-4}$ should be attained. The composite $\left(\bar{a}_{\text {eff }}\right)_{J}$ coefficients for Earth appearing in these results can be expanded as

$$
\frac{1}{m_{\oplus}}\left(\bar{a}_{\mathrm{eff}}^{\oplus}\right)^{J}=\left(0.54 \mathrm{GeV}^{-1}\right)\left[\left(a_{\mathrm{eff}}^{e}\right)^{J}+\left(a_{\mathrm{eff}}^{p}\right)^{J}+\left(a_{\mathrm{eff}}^{n}\right)^{J}\right],
$$

using $N^{e}=N^{p} \approx N^{n}=1.8 \times 10^{51}$ for Earth [25]. This implies sensitivity competitive with the maximum reach achieved to date on $\left(\bar{a}^{n}\right)_{J}$ and $\left(\bar{e}^{n}\right)_{J}[4]$ will be attained. Moreover, the constraints are on different combinations of coefficients than those involved in the existing tests [10, 26]. Thus combining results would yield additional independent sensitivities to Lorentz violation.

We note in passing that a full investigation of Lorentz violating couplings to spin in the presence of gravity may yield additional sensitivities to matter-sector coefficients associated with the test body; however, such an investigation is beyond the scope of the present work.

\section{CONVENTIONAL GRAVITOMAGNETIC EFFECTS}

The conventional General-Relativistic gravitomagnetic effect is more challenging to detect in anomalous spinprecession experiments for several reasons. However, it may in principle soon fall within the reach of these experiments. Presently, the largest gravitomagnetic effect on spins in the lab is that of Earth. Thus it is interesting to consider the effective $b_{\mu}$ due to the conventional gravitomagnetic field of Earth by inserting the appropriate $h^{0 j}$ into Eq. (6). By modeling Earth as a uniformly rotating sphere of radius $R_{\oplus}$, the angular momentum of Earth due to its rotation on its axis at angular speed $\omega$ is $\vec{J}_{\oplus}=\frac{2}{5} m R_{\oplus}^{2} \omega \hat{Z}$, and the relevant quantity for insertion in Eq. (6) can be written

$$
h_{\oplus}^{0 j}=\frac{2 G}{r^{3}} \epsilon_{j k l}\left(J_{\oplus}\right)_{k} x_{l} .
$$


This yields

$$
\begin{aligned}
& \left(b_{\mathrm{gm}, \oplus}\right)_{1}=-\frac{G}{2 r^{3}} J_{\oplus} \sin \chi \\
& \left(b_{\mathrm{gm}, \oplus}\right)_{2}=0 \\
& \left(b_{\mathrm{gm}, \oplus}\right)_{3}=-\frac{G}{r^{3}} J_{\oplus} \cos \chi
\end{aligned}
$$

for the explicit form of $\left(b_{\mathrm{gm}, \oplus}\right)_{j}$, which is of order $10^{-38} \mathrm{GeV}$ on the surface of the Earth.

Although the effect lies within about 2 orders of magnitude of the expected sensitivity of the next generation of experiments, an observation of the effect would be challenging even if the required sensitivity were reached. At present, the relevant experiments are performed in the rotating reference frame of the Earth where an effective $b_{\mu}$ with components

$$
\begin{aligned}
\left(b_{\mathrm{nf}}\right)_{1} & =-\frac{1}{2} \omega \sin \chi \\
\left(b_{\mathrm{nf}}\right)_{2} & =0 \\
\left(b_{\mathrm{nf}}\right)_{3} & =\frac{1}{2} \omega \cos \chi
\end{aligned}
$$

arises due to noninertial frame gyroscopic effects. Note that the effect in Eq. (18) has components in the same direction as the larger contributions in Eq. (19). The effects could in principle be distinguished by their different dependencies on $r$, but doing so would likely be challenging in practice. Another approach would be to perform an anomalous spin-precession experiment in space, as has been suggested in the context of detecting curvature components [27]. Here a frame could be chosen that breaks the symmetry between Eq. (18) and Eq. (19) and/or the $r$ dependence of Eq. (18) could be better exploited.

Though their size lies well below the expected sensitivity of anomalous spin-precession experiments in the near term, it is worth commenting on conventional gravitomagnetic effects arising from the Sun and rotating masses in the laboratory. Following the same procedure leading to Eq. (18), one can obtain an effective $b_{\mu}$ for the gravitomagnetic effect of the Sun, which can be written

$$
\left(b_{\mathrm{gm}, \odot}\right)_{J}=-\frac{1}{2} G\left[\frac{3\left(J_{\odot}\right)_{K} x_{K} x_{J}}{r^{5}}-\frac{\left(J_{\odot}\right)_{J}}{r^{3}}\right] .
$$

Although about 6 orders of magnitude smaller than $\left(b_{\mathrm{gm}, \oplus}\right)_{j}$ contributions, $\left(b_{\mathrm{gm}, \odot}\right)_{J}$ has time dependence in the lab that is similar to Lorentz violation and decoupled from the noninertial frame effects. If the rotation axis of the Sun were exactly perpendicular to the ecliptic, the conventional gravitomagnetic effects due to the Sun in Earth-based anomalous spin-precession experiments could be obtained by inserting the simple constant expressions $\left(b_{\mathrm{gm}, \odot}\right)_{Y} \approx-\frac{G}{2 R_{\mathrm{ES}}^{3}} J_{\odot} \sin \eta$ and $\left(b_{\mathrm{gm}, \odot}\right)_{Z} \approx$ $\frac{G}{2 R_{\mathrm{ES}}^{3}} J_{\odot} \cos \eta$ into Eq. (11). Here $R_{\mathrm{ES}}$ is the Earth-Sun distance, $\eta$ is the angle of the $X Y$ plane relative to the ecliptic, and a circular orbit has been assumed.

A more detained analysis taking into account the inclination of the rotation axis of the Sun at an angle $i=7.25^{\circ}$ relative to the normal to the ecliptic yields

$$
\begin{aligned}
& \left(b_{\mathrm{gm}, \odot}\right)_{X}=\frac{-G J_{\odot}}{4 R_{\mathrm{ES}}^{3}} \sin i \\
& \times[\cos \Theta(3 \cos 2 \Omega T-1)+3 \sin \Theta \sin 2 \Omega T] \\
& \left(b_{\mathrm{gm}, \odot}\right)_{Y}=\frac{-G J_{\odot}}{2 R_{\mathrm{ES}}^{3}} \sin \eta \cos i+\frac{G J_{\odot}}{4 R_{\mathrm{ES}}^{3}} \cos \eta \sin i \\
& \times[\sin \Theta(3 \cos 2 \Omega T-1)+3 \cos \Theta \sin 2 \Omega T] \\
& \left(b_{\mathrm{gm}, \odot}\right)_{Z}=\frac{G J_{\odot}}{2 R_{\mathrm{ES}}^{3}} \cos \eta \cos i+\frac{G J_{\odot}}{4 R_{\mathrm{ES}}^{3}} \sin \eta \sin i \\
& \quad \times[\sin \Theta(3 \cos 2 \Omega T-1)+3 \cos \Theta \sin 2 \Omega T] .
\end{aligned}
$$

Here $\Omega$ is the angular speed of Earth along its orbit, and $\Theta \approx 14^{\circ}$ is the present angular distance along the ecliptic from the projection of the solar spin to the vernal equinox [28]. The interesting time dependence at the annual frequency arises from the first term in Eq. (20) and is due to the motion of the experiment through the solar gravitomagnetic field. These annual variations along with the radial dependence could help distinguish these effects from Lorentz violation.

The possibility of large angular momenta in the lab has also improved in recent years due to interest in high rotation-rate flywheels for energy storage. Though the effective $b_{\mu}$ that could be generated by such devices in anomalous spin-precession experiments is at least 8 orders of magnitude smaller than $\left(b_{\mathrm{gm}, \oplus}\right)_{j}$, the effect could easily be controlled and modulated allowing easy separation from other effects. From the stand point of SME-based tests of Lorentz symmetry, these systems would also offer knowledge and control of the source composition. There are also Lorentz-violating contributions to $h^{0 j}$ that lie at the same post-newtonian order as the conventional General-Relativistic gravitomagnetic effects, and an investigation of their implications may become interesting if sufficient sensitivity is attained in anomalous spin-precession experiments.

\section{SUMMARY}

In this work we have seen the unexpected result that experiments searching for anomalous precessions of intrinsic spins, which were designed to test Lorentz symmetry in a nongravitational context, have the ability to place new constraints on Lorentz-violating effects via gravitomagnetism. New constraints are placed on the SME coefficients for Lorentz violation $\bar{s}_{\mu \nu}$ and $\left(\bar{a}_{\text {eff }}\right)_{\mu}$ using existing data, and the next generation of these experiments is expected to yield sensitivities competitive with the best existing sensitivities to coefficients of this type. We also find that these experiments may one day observe the conventional gravitomagnetic effect on intrinsic spin. This demonstrates the continuing impact of Lorentz violation searches on the experimental investigation of fundamental physics. 
[1] V.A. Kostelecký and S. Samuel, Phys. Rev. D 39, 683 (1989); V.A. Kostelecký and R. Potting, Nucl. Phys. B 359, 545 (1991).

[2] D. Colladay and V.A. Kostelecký, Phys. Rev. D 55, 6760 (1997); Phys. Rev. D 58, 116002 (1998).

[3] V.A. Kostelecký, Phys. Rev. D 69, 105009 (2004).

[4] Data Tables for Lorentz and CPT Violation, V.A. Kostelecký and N. Russell, 2012 edition, arXiv:0801.0287 v5.

[5] R. Bluhm and V.A. Kostelecký, Phys. Rev. Lett. 84, 1381 (2000).

[6] V.A. Kostelecký and C.D. Lane, Phys. Rev. D 60, 116010 (1999).

[7] J.M. Brown, S.J. Smullin, T.W. Kornack, and M.V. Romalis, Phys. Rev. Lett. 105, 151604 (2010).

[8] B.R. Heckel, E.G. Adelberger, C.E. Cramer, T.S. Cook, S. Schlamminger, and U. Schmidt, Phys. Rev. D 78, 092006 (2008).

[9] V.A. Kostelecký, N. Russell, and J.D. Tasson, Phys. Rev. Lett. 100, 111102 (2008).

[10] V.A. Kostelecký, and J.D. Tasson, Phys. Rev. D 83, 016013 (2011).

[11] H. Thirring, Phys. Z. 19, 33 (1918).

[12] J. Lens and H. Thirring, Phys. Z. 19, 156 (1918); B. Mashhoon, F.W. Hehl, and D.S. Theiss, Gen. Rel. Grav. 16, 711 (1984).

[13] A. Papapetrou, Proc. Roy. Soc. (London) A209, 248 (1951); L.I. Schiff, Phys. Rev. Lett. 4, 215 (1960).

[14] L.H. Thomas, Phil. Mag. 3, 1 (1927).

[15] Q.G. Bailey, Phys. Rev. D 82, 065012 (2010).

[16] C.W.F. Everitt et al., Phys. Rev. Lett. 106, 221101
(2011).

[17] Q.G. Bailey and V.A. Kostelecký, Phys. Rev. D 74, 045001 (2006).

[18] V.A. Kostelecký, and J.D. Tasson, Phys. Rev. Lett. 102, 010402 (2009).

[19] R. Colella, A.W. Overhauser, and S.A. Werner, Phys. Rev. Lett. 34, 1472 (1975).

[20] R.J. Adler, P. Chen, and E. Varani, Phys. Rev. D 85, 025016 (2012).

[21] C. Gemmel et al., Phys. Rev. D 82, 111901 (R) (2010).

[22] L.-S. Hou, W.-T. Ni, and Y.-C.M. Li, Phys. Rev. Lett. 90, 201101 (2003); M.A. Humphrey et al., Phys. Rev. A 68, 063807 (2003); F. Canè et al., Phys. Rev. Lett. 93, 230801 (2004).

[23] V.A. Kostelecký and M. Mewes, Phys. Rev. D 66, 056005 (2002).

[24] J.M. Brown, private communication (numbers); Ref. 7] (ploted results).

[25] C.J. Allègre, J.-P. Poirier, E. Humler, and A.W. Hofmann, Earth Planet. Sci. Lett. 134, 515 (1995).

[26] H. Panjwani, L. Carbone, and C.C. Speake, in V.A. Kostelecký, ed., CPT and Lorentz Symmetry V World Scientific, Singapore 2011; M.A. Hohensee, S. Chu, A. Peters, and H. Muller, Phys. Rev. Lett. 106, 151102 (2011).

[27] S. Mohanty, B. Mukhopadhyay, and A.R. Prasanna, Phys. Rev. D 65, 122001 (2002).

[28] W.M. Smart, Textbook on Spherical Astronomy, (Cambridge, London, 1977). 